\title{
Product-Lining the Elinvar WealthTech Microservice Platform
}

\author{
Marcus Pinnecke \\ Elinvar $\mathrm{GmbH}$ \\ Berlin, Germany \\ marcus.pinnecke@elinvar.de
}

\begin{abstract}
Software product lining is the act of providing different but related software products under the same brand, known as a software product line (SPL). As engineering, management and validation of SPLs is far from trivial, special solutions for software product line engineering (SPLE) have a continuous momentum in both academic and industry. In general, it is hard to judge when to reasonably favor SPLE over alternative solutions that are more common in the industry. In this paper, we illustrate how we as Elinvar manage variability within our WealthTech Platform as a Service (PaaS) at different granularity levels, and discuss methods for SPLE in this context. More in detail, we share our techniques and concepts to address configuration management, and show how we manage a single microservice SPL including inter-service communication. Finally, we provide insights into platform solutions by means of packages for our clients. We end with a discussion on SPLE techniques in context of service SPLs and our packaging strategy. We conclude that while we are good to go with industry-standard approaches for microservice SPLs, the variability modeling and analysis advantages within SPLE is promising for our packaging strategy.
\end{abstract}

\section{CCS CONCEPTS}

- Software and its engineering $\rightarrow$ Software design engineering; • Computer systems organization $\rightarrow$ Cloud computing; - Applied computing $\rightarrow$ Event-driven architectures; Serviceoriented architectures.

\section{KEYWORDS}

product families, configuration management, variability management, technologies and concepts, microservice platforms

ACM Reference Format:

Marcus Pinnecke. 2021. Product-Lining the Elinvar WealthTech Microservice Platform. In 25th ACM International Systems and Software Product Line Conference - Volume B (SPLC '21), September 6-11, 2021, Leicester, United Kingdom. ACM, New York, NY, USA, 9 pages. https://doi.org/10.1145/3461002. 3473947

\section{INTRODUCTION}

A software product line (SPL) is a group of similar software products that base on a common code but differ in terms of exposed

Permission to make digital or hard copies of part or all of this work for personal or classroom use is granted without fee provided that copies are not made or distributed for profit or commercial advantage and that copies bear this notice and the full citation on the first page. Copyrights for third-party components of this work must be honored For all other uses, contact the owner/author(s).

SPLC '21, September 6-11, 2021, Leicester, United Kingdom

(c) 2021 Copyright held by the owner/author(s).

ACM ISBN 978-1-4503-8470-4/21/09.

https://doi.org/10.1145/3461002.3473947 features [42]. A feature in context of SPL might be any kind of observable behavior of a software or a related artifact that is expected by a user for the particular domain of that software [4, 23, 42]. Features may stem from upfront domain analysis [23], are manually reverse engineered from the code base by some description [46], or without such description [26], to name a few origins of features in SPLs. However, features are typically scattered across the code base as they implement cross-cutting concerns that do not naturally map to components, classes or modules in traditional programming paradigm, or even span over applications and databases [3, 33]. In any case, features might be activated for some products and deactivated for others, which effectively defines the SPL [42]. For several decades, academic is arguing for and researching on software product line engineering (SPLE) that distinguishes explicitly between domain engineering and application engineering. The former results in an explicit formal variability model, often called feature model, that is used to express features, their relationships by means of constraints, and that is the base for advanced analysis and code generation $[4,5,7,17,21,23,30,40]$. The latter, application engineering, aims to enable and support feature-aware programming that results in building blocks for code generating [4, 42]. In 2014 Thüm et al. argued that SPLs analysis is far from trivial as it is computational expensive: the small number of 33 independent, optional features alone lead to a SPL with more possible products as people on earth [42]. With this observation in mind, it surprises that SPLE techniques, such the paradigm of Feature-Oriented Programming (FOP), still seem to wait for their moment to shine across industries. To close this gap, we aim to provide insights into our practice, judge SPLE under different use cases, and elaborate where we think it will shine for us.

In this paper, we provide insights in product lining the Elinvar WealthTech PaaS, and explain where variability occurs and how we manage it. We start with the introduction of our platform in Section 2. Then, we consider:

- Configuration Management enabling us to statically or dynamically configure our services at compile-time, boot-time, or runtime-time (Section 3), and

- Product Lining the Platform in which we show how we design, implement and test service product lines, and which packaging concepts exists.

Finally, we discuss whether SPLE could be a good fit for us, the benefits of SPLE and its costs and risks for microservice product lines and our packaging strategy in Section 5. We end with a summary in Section 6 and list references in Section 6.

Contributions. We aim to contribute a practical point of view on software product lining and variability management by example of our own platform under several perspectives, and provide our judgment where we believe SPLE will shine in our company. 
All mentioned websites were last accessed 2021/06/11. Finally, please note that both Elinvar as a company and the Elinvar WealthTech PaaS are constantly developing and growing, so any fact shared about us as a company, or about our solutions is subject to change and represents the current state as of June 2021.

\section{THE ELINVAR WEALTHTECH PLATFORM AS A SERVICE}

Elinvar ${ }^{1}$ is a BaFin-licensed WealthTech Platform as a Service, enabling asset and wealth managers to digitalize their business models. The multi-tenant solution offers a broad range of service packages for core processes along the entire value chain of asset and wealth management as well as third party connections. Cloud-native and developed for B2B2X, Elinvar aims to connect the entire wealth management ecosystem on one highly scalable PaaS. As an enabler of digitalization and wealth management-as-a-service, Elinvar offers established companies in asset and wealth management opportunities to execute new business models, access new value chains and optimize their costs. Elinvar is constantly growing and unites as of now more than 130 team members from 35+ nationalities. 70\% of the team are working in directly tech-related roles, assigned to specific departments including eight engineering squads.

\subsection{Technology Stack}

Today, our platform runs on more than one hundred microservices with a total sum of approx. 842k lines of Java code, $371 \mathrm{k}$ lines of SQL, 168k lines of JSON, 58k lines of YAML, 43k lines of XML, and other languages such as XDS, Python, Markdown, Bash code, JavaScript, Dockerfiles.

Our platform is a cloud service running on $\mathrm{AWS}^{2}$ with Kubernetes ${ }^{3}$ and Docker ${ }^{4}$. For monitoring, we use industry standard tools like Grafana ${ }^{5}$, Opsgenie ${ }^{6}$, and Prometheus ${ }^{7}$. Typically, our backend services are written in Java and store their states in PostgreSQL ${ }^{8}$ databases, although exceptions exist here. We use Apache Kafka ${ }^{9}$ as our primary distributed and persistent database and message broker on which we build an event-oriented architecture. Besides pure fire-and-forget event production, we support remote procedure calls implemented over Kafka. However, all backend services benefit from industry standard configuration management and the programming model powered by the Spring ${ }^{10}$ Framework with dependency injection that are enriched by in-house core libraries that expose reusable components across individual services. The frontend runs on Node.js ${ }^{11}$ with the React ${ }^{12}$ JavaScript Framework enabling us to build progressive web applications.

\footnotetext{
${ }^{1}$ Elinvar: https://www.elinvar.co.uk

${ }^{2}$ Amazon AWS: https://aws.amazon.com/de/

${ }^{3}$ Kubernetes: https://www.kubernetes.io

${ }^{4}$ Docker: https://www.docker.com

${ }^{5}$ Grafana Labs: https://grafana.com

${ }^{6}$ Atlassian Opsgenie: https://www.atlassian.com/de/software/opsgenie

${ }^{7}$ Prometheus: https://www.prometheus.io

${ }^{8}$ PostgreSQL: https://www.postgresql.org

${ }^{9}$ Apache Kafka: https://kafka.apache.org

${ }^{10}$ Spring: https://spring.io/projects/spring-framework

${ }^{11}$ Node.js: https://nodejs.org/en/

${ }^{12}$ React: https://reactjs.org
}

\subsection{Microservice Classes}

At Elinvar, we run broadly three classes of microservices: businesslogic services, infrastructure-services, and platform-test services.

Business-Logic Services. The first class, business-logic services, consists of services that implement our business logic. Services belonging to this class actually implement the solutions that we provide to our clients. In this class, we have services for order management, data migration, market data provisioning, anti-fraud detection, reporting, or portfolio management, to name a few. Depending on the selected platform package, a particular subset of these services will be provided to our clients. Some services are mandatory, such as customer relationship management, while other services are optional, such as anti-fraud detection. In addition, some solutions require multiple services to be contained. We will discuss platform packaging more in detail in Section 4.3.

Infrastructure Services. The second class, infrastructure-services, is dedicated to support our infrastructure, including two-factor authentication and single sign-on, message inspection, and more. Most notably in the context of this paper, we developed an in-house service called the Configuration Services (CS). The purpose of the $\mathrm{CS}$ is to enable dynamic configuration of services either globally or for particular clients. Without the CS, we would either require a re-deployment of the affected services as configuration files are part of docker images, or require manual re-configuration of the Kubernetes pod configuration. However, we partially enable administrators of our clients to configure certain parts of our platform according to their individual preferences and needs. We will provide more details of the CS in Section 3.4.2.

Platform Test Services. The last class, platform-test services, are a specialization of our services responsible to integrate third party services or to communicate with external partners. We deploy our platform following a continuous integration / continuous delivery up to multiple times a day to different deployment environments. Each of these environments serves a particular purpose with increasing access restrictions. For development and testing purposes, we run two dedicated environments in which third party services and external partners are not connected. For these two environments, we provide dedicated mock services that effectively help us to simulate the behavior of external services and partners.

\section{CONFIGURATION MANAGEMENT}

For the majority of our services, we have some kind of configuration, which enables us to configure the service or parts of it according to the actual needs, the environment, or client-related properties.

Configuration management in our context is a structured process, backed by technology, to configure particular parts of our platform. Examples of these parts are feature flags that conditionally turn on or off a certain functionality, transport and connection strings to define which message topics are read and written by a service or what authentication parameters are used to access a network storage server, or particular settings affecting internal processing logic, $\log$ creation, or other behavior. It is worth to note that we do not use version control systems for our configuration management, as we do organize (purely code-based) variants by means of multimodule projects rather than in dedicated branches. Although we 
are used to maintain service variants in dedicated branches, most notably for hotfixes, we use version control primarily to merge changes to the code base delivered by parallel and independently working developers in a control manner into our master branch. Master branches are regularly tagged and developed. We give more details on this subject in Section 4.2.

In the following, we show the most used techniques to support our configuration management, namely object dependency resolution in Section 3.1, compile-time configuration definition with evaluation at service boot time in Section 3.2 and with evaluation at compile time in Section 3.3, as well as runtime-definition with evaluation at runtime in Section 3.4.

\subsection{Dependency Injection}

Dependency Injection (DI) is a design pattern that enables us to resolve dependencies from one object to another at runtime without the need to manually maintain another factory object [36]. We use DI to replace implementations of particular components depending on certain context: For instance, one of our in-house libraries exposes an abstraction over our message broker that we use for inter-service communication. In the context of integration tests, we typically set the implementation to a mock which does not require Kafka to be present. Such mocks enable us to test certain conditions, such as the publication of a particular message.

There is a variety of tools enabling DI. We initially used to utilize Google Guice for this purpose but recently migrated to the native DI support in the Spring Framework. Considering that both, Guice and Spring, provide reasonable functionalities for our needs, we decided for this migration in order to no longer use two different tools, where one is fully sufficient.

Despite the use for DI in the context of unit and integration testing, we make intense use of DI for designing service families as we will explain more in detail in Section 4.2.

\subsection{Static Configuration}

By static configuration we mean a set of options and values - that can vary in principle, but are fixed to certain values when an executable is built - and service states. A miss-configuration would be detected already during service boot. In the following, we describe static configuration files, state management, and feature code.

3.2.1 Configuration Files. To avoid mixing configuration concerns with productive code, we maintain one or more configuration files per service that spatially collect these configurations. As we are using configuration files, static configuration and dynamic configuration (Section 3.4.2) pass over fluently into each other.

For us, the difference between static configuration and dynamic configuration in the context of configuration files is purely dependant on whether we allow a particular value to be overwritten by some environment variable during load time of the service. As we are talking more about dynamic configuration in a dedicated section, we will now focus more on configuration file values that do not change during load time.

The most prominent example for static configurations are the pre-defined topic names for our message broker which are used by a particular service. Whenever a service subscribes or publishes to an event stream topic or table topic, the name of that topic is contained in the configuration file. Based on this, we likewise define which entity class models the data that are read from esp. written to that topic, potentially set some topic-scoped options (e.g., the policy for offset committing), and use these informations for code generation. The latter enables us to use this structured textual configuration data natively and in a type-safe fashion within Java. We base our service configuration files primarily on the Spring Framework.

3.2.2 Database States. As state is part of the configuration management, we further store database migration scripts per-service as a static asset. Database migration enables us to keep a consistent database schema across local test beds and all deployment environments. We require migration scripts to be an ordered list of modifications to the database structure, so we can guarantee that no database is inconsistent in terms of its structure and schema. This benefit of consistent databases outweighs in our view rare cases of conflicting rollbacks. We use Flyway ${ }^{13}$ as our solution to express database migration and schema evolution.

3.2.3 Feature Code. Finally, we have some rare cases where conditionally executable feature-related code artifacts are stored as static asset. Typically, we try to avoid code that is conditionally executed by some runtime-related value as we are structuring feature code by means of modules and components that is bound via DI during compilation time of a particular service (see more in 4.2). Next, we describe one case for runtime-configurable feature code.

During movement of functionality from one service into another, we follow the strategy that both the original service and the destination service can execute the functionality, but a dynamic configuration option decides which services actually perform the logic. This is reasonable as the original service is a reliable and easy-to-activate fallback in case the destination service shows some unintended behavior. Once a reliable take-over of the functionality by the destination service is possible, the functionality code in the original service is removed.

We use a mixture of concepts for conditional feature code execution. The code is written in Java, registered and (de)activated either via configuration files and environment variables during service boot, or registered and (de)activated via our Configuration Service (cf. Section 4.2) at runtime.

\subsection{Compile-Time Configuration}

At Elinvar, compile-time configuration is a special form of static configuration (see Section 3.2). In contrast to the static configuration, compile-time configuration will stop the compilation process when a miss-configuration is detected. We achieve this by generating Java code out of some configuration description, such as configuration files or a novel markup language for our alerting system. Currently, we support compile-time configuration for definitions of and configuration for service topics, imported or exported remote procedure call definitions, and alerting.

For topics, each service uses a configuration file to describe which topic it will publish to resp. subscribe to. In addition, the configuration per-topic include which Java class to use for serialization/deserialization into the message wireformat, JSON. Finally, some options can be defined such as the Kafka offset commit policy.

\footnotetext{
${ }^{13}$ Flyway: https://www.flywaydb.org
} 
With this information, our build automation will generate proper code that enables type-safe integration into the Java code of our services. In case of a miss-configuration, such as specifying an unknown message class name, the subsequent compilation process will fail as the Java compiler is not aware of that class name. For remote procedure call definitions, we proceed similarly.

Alerting at Elinvar is raising a notification to a particular group of addressees about an issue within a service context that potentially requires manual correction. For instance, an alert is raised with the responsible developer team as addressee whenever one of their services cannot properly process incoming messages from a third party service (cf. [14]). An alert has an unique identifier, a particular severity level, belongs to a particular alert group, contains a summary and message, further hints, and more. We designed a custom markup language, AlertML, which is specialized to express these informations in a compact and structured way. Based on perservice AlertML files, we generate Java code so that alerts can be instantiated, variables within information are bound properly, and alerts are raised in an unified and less noisy way. As for definitions of topic usage and remote procedure calls, the build automation supports constantly well-defined alerts, as giving notice whenever an unknown severity level is used, or when a variable was defined but not bound in the AlertML file.

\subsection{Dynamic Configuration}

At Elinvar, dynamic configuration is verification, modification and application of configuration options during the lifetime of a service (cf. [15]). The most simple case is that a configuration file may expose a configurable value, such as a particular threshold to trigger some alerting, as an environment variable which can be set before the service startup (cf., Section 3.2.1). There are two kinds of dynamic configurations, load time configuration and life-time configuration. As we strongly aim to avoid service downtimes, we favor life-time configuration before load time configuration whenever possible and reasonable. However, we present both concepts.

3.4.1 Load Time Configuration. Load time configuration enables to modify an option before the service starts, is read and applied after verification by the service, and stays static until the end of the service lifetime. The service will controlled stop booting and raise a proper alert in case that a verification issue, such as providing unsupported values, is detected. For scenarios which allow to reboot the service in order to apply a configuration change, load time configuration is a reasonable option. In scenarios which require a constantly running service, we preferably use our Configuration Service (cf. 3.4.2).

Environment Variables. Whenever a service is configurable via some environment variables, these environment variables are set during deployment of the service into some environment.

We use Kubernetes configuration per service and per environment to set environment variables. Typically, we use environment variables to define Kafka-related values, to define internal and external routing, to pass parameters to the JVM, to configure cron expressions for internal scheduling, to enable or to disable alerting rules, to define which character sets are used, to setup time window lengths for data migrations, and for many more tasks. For confidential data, we link to secrets such as for PostgreSQL database connection parameters, for Amazon S3 Storage Servers, for internal service authentication, for SFTP connection parameters, or for external services and partners. We further define filter conditions on incoming messages to configure a particular service within a service family to be responsible for a particular subset of messages. For instance, with such filter conditions we define that the Order Gateway in variant $A$ is responsible for messages related to the custodian bank $A$ while other variants in the service family will ignore messages for $A$ completely. We will describe this process more in detail in Section 4.2.

In case we have feature code in place as described in Section 3.2.3, we typically enable or disable that code via a feature toggle bound to an environment variable. For feature code we typically do not use life-time configuration (cf. Section 3.4.2.

ConfigMaps. Kubernetes provides a technique called ConfigMaps ${ }^{14}$ that enables us to define configuration options which then are either passed as environment variable or startup arguments, or which can be mounted as a file into a volume.

We use ConfigMaps to populate Kafka bootstrap servers to our services, to connect services to the application performance manager Glowroot, or to mount vendor-specific configuration files. Examples for the latter include special purpose database storage using Amazon RDS, advanced searching and analysis via ElasticSearch, log forwarding using Firebeat, visual analysis using Kibana, data collection and transformation using LogStash, monitoring using Prometheus, and more. In addition, we pass bash scripts whenever needed via ConfigMaps.

3.4.2 Life-Time Configuration. Life-time configuration is similar to load time configuration but is responsive to changes in the configuration without the need to reboot a service. In contrast to load time configuration, services continue running when a verification issue, such as providing unsupported values, is detected. However, services will reject the updated configuration, continuing with the former configuration, and raising proper alerts to the operations department. Life-time configuration enables us to dynamically reassign client-service association, to synchronize service-local states with platform-global states, or to dynamically modify processing activities, to name a few.

Distributed State. Stateful services within our platform maintain their own states typically in a database attached to these services. However, the state of the entire platform is stored in a distributed and durable database, Kafka. As a service communicates by messages sent over certain Kafka topics, other services may read these messages and update their internal states if needed. Beside message topics, we maintain table topics which define the latest shape of an entity. For instance, we store client information and custodian bank associations as table topics. With this approach in combination with filtering as described in Section 3.4.1, a service instance is able to decide which topics are of interest and for which clients or custodian banks the instance is responsible for. What rules should apply here, is subject to the service specification as described in

\footnotetext{
${ }^{14}$ Kubernetes ConfigMaps: https://kubernetes.io/docs/concepts/configuration/ configmap/
} 
Section 4.2.1. However, updating the internal state with the external distributed state is typically done whenever needed during the lifetime of a service and does not require service reboots as affected components within the service are updated dynamically.

Configuration Service. At Elinvar, we developed a dedicated service, called the Configuration Service (CS), that enables life-time configuration of services by administrators. The CS is a mediating service which registers, stores, deploys, imports and exports configuration, and runs basic type validation. For more advanced validation that requires some semantic checks, the CS delegates validation to the owning service. The CS is build upon the concepts of configuration, schema, and deployment. A configuration, in the context of the CS, is a list of key/value properties with a formal schema that represents property descriptions and property types as well as basic per-property constraints, such as a non-empty constraint to strings or signing for integers. Whenever more complex constraints are needed, such as constraints between properties or constraints that require computations, these validations are delegated to the responsible service before a configuration deployment is made. After successful validation, a deployment is then an immutable application of a particular configuration. Besides having a centralized versioned repository for configurations, the CS enables the automatic generation of user interface forms to modify configurations and their properties.

Technically, the CS makes use of distributed state (cf. Section 3.4.2) to publish validation request messages for every new configuration. A request is picked up by the owning service using techniques such as filtering as described in Section 3.4.1. The validation outcome is then published as a message to a pre-defined topic the CS is subscribing to. In case of a successful validation followed by a deployment action by an user, the CS publishes a configuration update message to the effected service(s). These updates are picked up by the service, and typically directly applied. It is worth to note that the CS does neither analyze nor manage the variability within the configurations it provides. In particular, the CS will neither give any statement whether a particular configuration option will have any effect at all [34], nor hide options that will not be used due to the selection of other options [42], to name a few restrictions. By our current company policy, the service developers and product managers are in charge to properly define reasonable and most easy-to-use configurations, and describe their behavior and side effects via a dedicated documentation.

\section{PRODUCT LINING THE PLATFORM}

Our platform provides service packages for core processes in asset and wealth management, such as customer relationship management, portfolio management, advisory, reporting and invoicing, online client access, communication, and many more. In addition, our platform is built to connect to services of third party providers. Some of our services, especially these who are connecting to third party services and partners, belong to a service family. A service family in our context describes services which have the same purpose but require some kind of specialization to fulfill that purpose.

In this section, we introduce a running example in Section 4.1, explain how we manage service families in Section 4.2, and finally explain our concepts for packaging multiple services in Section 4.3.

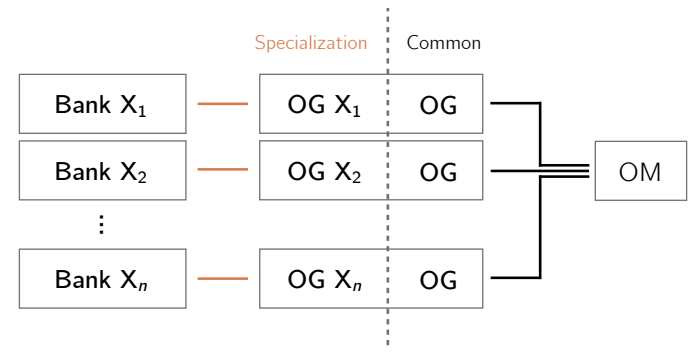

Figure 1: Order Gateway specialization towards banks, 2021.

\subsection{Running Example}

We will focus on service families as they are of central interest for product lining our platform, using the Order Gateway service family as an example in Section 4.1.1. To show how single-product services interact with service families, we will then introduce the Order Manager service in Section 4.1.2. In Figure 1, we show an illustration of the interaction between the Order Manager and the Order Gateway service family, which we will explain in the next two sections. Afterwards, to illustrate how we package our platform, we describe briefly two optional services, the AML third party service package and the Postident benefit in Section 4.1.3.

4.1.1 The Order Gateway Service Family. The Order Gateway (OG) is responsible for bidirectionally communication with custodian banks to forward order insertions or cancellations of requests from Elinvar to a custodian bank, and to forward order status updates from a custodian bank to Elinvar. An order stems from the Order Manager (OM) service which bundles orders calculated by a distinct service (cf. Section 4.1.2). Incoming order updates will be forwarded to the OM for further usage in our platform, such as to update portfolio information, or to display order status information. Since each custodian bank defines their own communication protocol (e.g., message format, rules or network protocol), the OG is a service family having a common interface into our platform and a specialization for the particular custodian bank.

4.1.2 The Order Manager Service. The Order Manager (OM) service is responsible to forward order batches to the OG so that bankspecific orders are actually placed. An order batch is computed in the OM by aggregation of buy and sell orders including price limits of all affected clients for the respective bank. Depending on the bank, the actual aggregation strategy can differ.

4.1.3 The AML Service Package and Postident Benefit. The Anti Money Laundering (AML) third party service package is used to detect criminal activities related to monetary income by checking that the respective assets are properly acquired according to laws, regulations, and other rules. Postident is a service by Deutsche Post AG to confirm personal identification, required to ensure compliance with the Money Laundering Act, obliging banks to identify their clients upfront before any account is opened. At Elinvar, we run a dedicated micro service that enables us to perform a Postident procedure. Both, the AML check and the personal identification, are optional for Elinvar clients to subscribe to. 


\subsection{Microservice Families}

The majority of our services which belong to certain families are developed and maintain in the department for third party integration, the Platform Connectivity (PLC). By design, PLC services show identical behaviour when communicating to internal services despite the specialization to external protocols of different partners and providers. Namely, the Order Gateway $\mathrm{OG}_{A}$ for custodian bank $A$ reads and publishes the same messages the $\mathrm{OG}_{B}$ for custodian bank $B$. Thus, $\mathrm{OG}_{A}$ and $\mathrm{OG}_{B}$ intersect in their behavior towards other services in our platform, and differ in their specialization towards $A$ resp. $B$. As incoming messages encode whether they belong to $A$ or to $B$ by using filtering, as described in Section 3.4.1, the $\mathrm{OG}_{A}$ gets active while $\mathrm{OG}_{B}$ ignores the message or vice versa.

4.2.1 Specifications. The variability inside a service family is specified by the owning product manager in conjunction with the responsible developer(s), and other team members from different departments. The specification is refined during development and continuously synchronized with the implementation bidirectional.

For synchronization from specification towards implementation, the product manager describes user stories that are picked up by developers. In the opposite direction, the developer is allowed to refine the specification in collaboration with the product manager (if needed). In any case, communication between the involved parties is one key factor to keep specification and implementation in sync. Besides organizational-driven methods, we have technical-driven methods in place. For instance, for services that expose a REST API, we specify the interface via Swagger files. Another example is the implementation-independent definition of alerts with Alert ML.

However, by example of the OG there is a specification that defines the behavior of all OGs towards other services, and for each bank $X$ there is a dedicated specification defining $\mathrm{OG}_{X}$. Typically, a specification document describes the business logic of a service, input and output messages in the big picture taking connected services into account, definitions and details to workflows in the service, as well as side effects, alert conditions, lists related tickets in our ticketing system, and more. As we are in control of the internal behavior and can define the specification towards the intended bank or third party provider, variability is implemented by proper industry-standard concepts and by good engineering.

4.2.2 Domain Artefacts. According to Pohl et al., domain artefacts include the product roadmap, the domain variability model, the domain requirements, the domain architecture, the realization artefacts and the test artefacts [35]. For the product roadmap, we typically maintain $n+1$ (versioned) specification documents for $n$ specializations and one specification document to define common behaviour. Market introduction plans are typically defined per specialization and supported by the application of the agile Minimum Viable Product (MVP) approach. MVP allows us to keep requirements and solutions close together over the course of iterations on implementations and specifications. The domain variability model for a service family is implicitly expressed by the specification documents on common behaviour and specialized behaviour, while it is explicitly expressed in a structured data model for service packages. We already discussed the first and will provide more details on the second in Section 4.3.
In the context of a service family, domain requirements for the common behaviour are defined internally and refined iteratively whenever a specialization requires it. Per specialization, domain requirements are primarily analyzed and maintained by project managers resp. product managers who work in close cooperation with the external partner to which we specialize. This procedure can result, for instance, in a specification document defining a well-defined mapping between external and internal protocols. The domain architecture, by the example of PLC services, is typically designed as an abstract service that leaves specialization-specific interfaces up to the implementation of a specialization. A specialization then implements these interfaces and has some specializationspecific architecture driven by the need to manage the connection to external partners reliably. Such specific architecture details might include specific state control mechanisms, error handling, or partner-specific alerting, to name a few. A specialization, therefore, inherents the variability of the abstract service and enriches it with its specifics. Configuration management is then managed as described in Section 3.

Realization artefacts include (but are not limited to) hand-written or generated source code files, configuration files or configurable options, specification documents, deployment information, or build automation scripts. As stated in the previous paragraph, we distinguish between code to implement the abstract service and code to implement the specialization. From a dependency perspective, any specialization can access common code in the abstract service but specializations are unaware of each other. Shared code across services, such as the construction of an IBAN character string or base remote procedure functionality is located in one of the two inhouse libraries. The mentioned distinction between abstract service and specialization(s) is reflected by the per-service build automation script(s) that, among others, specify dependencies, define code generation, or jobs for publication into an internal code repository if needed. Finally, we follow an exhaustive test plan on several levels of a service life cycle in order to provide reliable and robust functionality. More precisely, we live the test-driven culture from a single unit (such as a single method) covered by industry-standard test frameworks up to automated testing across several running services in environments. As for the realization of artefacts, we focus on testing specific components such that we test the abstract service, each specialization on its own, and dedicated libraries independently from each other. However, where components are connected, we run integrations tests.

4.2.3 Implementation. Today, service families are organized as multi-module projects where one dedicated module, called Common, implements the behavior into our platform. The Common module defines interfaces which are then implemented by the specific specialization. For example, the OG Common handles filtering, monitoring, shared alerting and provides an interface, called Client, that must be implemented to specialize the OG for a bank.

Shared Libraries. Beside shared functionality in Common which is scoped to the service family, a service has access to two major in-house libraries which provide implementation for re-usable functionality, the Elinvar Core Library (ECL) and the Connectivity Commons Library (CoCo). At Elinvar, a typical service uses at least the ECL, while the majority of PLC services additionally depend on 
CoCo which exposes functionality for services connecting to third party providers. However, to implement a specification (cf. Section 4.2.1) we are using a subset of configuration techniques as described in Section 3. Specializations are encapsulated in dedicated modules which are fused by DI and Common to construct the final product.

Defect Handling. As module-specific variability and issues are scoped to the respective module, we can avoid major issues in practice. Doing continuous development with continuous integration, we usually detect defects early and typically must only deal with small increments. Clearly, a defect in Common would affect all specializations but should be perceived in one of the earliest deployment stages within our structured testing process. In contrast, a defect in a specialization is limited to the scope of that module, but should be detected early in the deployment process similar to Common.

Lightweight Libraries. Finally, beside sharing of Common code, ECL and CoCo with specializations, we plan to export message classes in a dedicated small module as an artifact to our internal Nexus Repository. The Common module has still ownership of message class definition without the need for a slower librarybased development process, and other services can directly use a particular (or latest) version of message classes read or written the service. We make use of this approach for our testing platform (cf. Section 2.2) but still have the priority on the productive service.

4.2.4 Builds, Tests and Deployments. For services and service families, we run a sophisticated build pipeline involving unit and integration tests, build servers, and continuous integration. Most important for testing is that we rely on DI to construct particular tests modules in which we run end-to-end integration tests which test the business logic functionality as specified. However, continuous integration involves automatic deployments to test environments without connection to third party, deployment to test environment with third party connected and finally deployment to the production environment in a dedicated process but this is out of scope of this paper. Services within a service family are deployed as standalone products tightly integrated into our platform ecosystem. However, for test environments in which no third party connection is available, we directly connect to platform test services as described in Section 2.2. With these platform test services, testers and product managers can test in addition to the developer tests that the services correctly integrate into the overall processing pipeline of our platform. While this testing is typically done manually when starting a new service project, automation tests, called umbrella tests, are added to guarantee modifications to a service do not break expected behavior. Please note that automation tests also cover frontend tests using the Cypress ${ }^{15}$ testing framework.

4.2.5 Inter-Service Communication. As we are exposing the same interface and message format across services in a service family, service-service communication is unaware of specialization, with the exception that control information in messages will delegate messages to particular services inside the service family. However, this control information is not artificial, but part of the business logic. Consider the OM as an example. Whenever an order batch

\footnotetext{
${ }^{15}$ Cypress: https://www.cypress.io
}

is sent to the OG service family, the related message necessarily contains the associated custodian bank, as this information is part of the data model. The custodian bank information is, however, the key at which a service inside the OG service family decides if that message must be processed or be ignored. Finally, as serviceservice communication is done via a persistent medium, Kafka, inspection is possible, as messages sent between services are durable and immutable. In combination with historical information stored within each service database, issues, if any, can be resolved and the root cause can be quickly found.

\subsection{Platform Packaging}

Beside custom branding, we provide our solutions by means of service packages. Conceptually, we define service packages over a set of benefits. We depict an illustration of service packages and benefits (as an excerpt) in Figure 2.

Benefits. A benefit is a solution provided to a client which is implemented within a particular micro service, while micro services may implement multiple benefits. Benefits enable us to present our solutions on a more fine-grained scale, thus making them more visible for our potential and existing business partners. As of today, we provide nearly one hundred dedicated benefits, such as supported client onboarding, order analysis, whitelist with research opinion, or the implementation of PostIdent (cf. 4.1.3). Benefits belong to a particular class, such as feature, and a benefit belongs to a particular group, such as end client identification. Finally, the majority of benefits are reflected in some user interface inside the frontend, and have a human-readable textual description.

Service Packages. A pre-defined selection of benefits forms a service package. Please note that benefits may overlap given to packages such that packages are not a partitioning of the set of benefits. By offering more than fifteen Elinvar-owned service packages as well as the connectivity to several service packages by third parties, we support a broad range of target operation models, including private and universal banks, independent discretionary portfolio managers, insurance companies, and more. To illustrate, the portfolio management service package includes the benefits of core portfolio management, rebalance log, fund-look-through, provision of historical prices, order analysis, and more. Beside contractual advantages, service packages enable Elinvar as a company to have a cleaner understanding of service packages as independent units, improving the internal ownership overview and enhancing our organizational structures.

\section{DISCUSSION}

It is important to understand that SPL naturally occur once a software product must be specialized to more than one target (cf. [6, 30, 45]); might it be slightly different database schemata for two or more different clients, or for technical reasons, such as compilation to different machine architectures as regularly being done for the Linux kernel [19]. Therefore, any technique that enable such specialization can be considered as a technique to craft an SPL, such as code cloning [38, 39], configuration management [32], annotation-based approaches like code pre-processing and composition-based approaches like libraries, plug-ins and frameworks [4], 


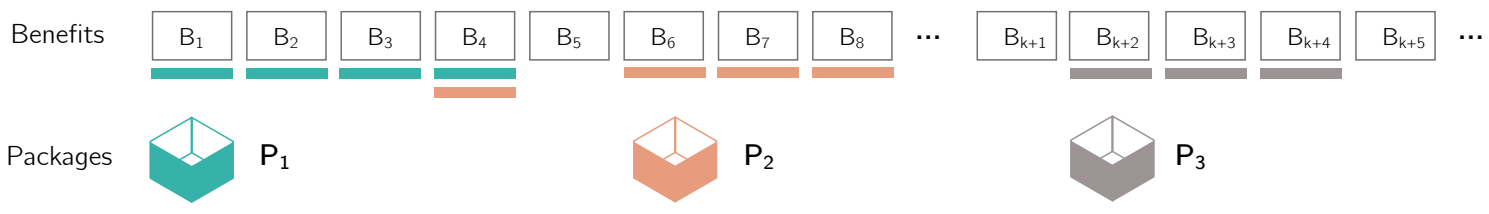

Figure 2: Service packages and benefits, 2021.

or particular programming paradigm like Feature Oriented Programming (FOP) with tools like AHEAD [8].

\subsection{Software Product Line Engineering}

For more than two decades (cf., [16]), academic is arguing for a more structured and more controlled approach addressing challenges with SPLs, software product line engineering (SPLE). SPLE emphasizes variability management within and between software by adding an explicit formal model, typically called feature model [4, 37], which states features and dependencies between them, and can be used to organize feature-related code orthogonal to the actual programming paradigm. In its consequence, software product lining in SPLE is divided into two distinct activities, domain engineering in which variability is modeled purely formally by means of a feature model, and application engineering in which the actual code is written under a paradigm similar to FOP. One main challenge addressed by SPLE according to [30,42] is guaranteeing correctness and reliability of the entire product line, which means across all possible products within SPL and not only within the subset of products in actual use. This guarantee is based on type checking, static analysis, model checking, and theorem proving the SPL. The variability inside a SPL is typically described by a formal specification and is carried out by analyses based on products, based on the product family, based on features, or a combination of the former [9-11, 41]. SPLE inherently requires the variability model and integration of feature-oriented programming capabilities into non-feature-oriented languages. Hence, advanced tooling to make SPLE practical usable is needed [2, 13, 20, 24, 27]. For example, FeatureHouse [5] extends several languages with composition-based concepts effectively transpiling the result when needed to the original language via code generation. Another example is the Eclipse extension Feature IDE that combines the FOP paradigm with modeling, type checking composition and testing capabilities [1, 25, 31, 43]

\subsection{Microservice SPLs}

At Elinvar, we do not strongly rely on a formal feature model for service variability management, as we aim to keep our services lean and simple. As pointed out in Section 3.2.3, we try to avoid feature code within a service and aim for a robust implementation of the business logic with industry-standard languages and tools. Ideally, a service would implement only one feature, which, however, is hard to guarantee as evolution in services tends to consolidate related features in a single service. If a service exceeds a particular complexity, we typically start to split this service. From our practical point of view, SPLE solves a relevant challenge, namely guaranteeing on all possible products within the product line. As of now, this is not relevant for us to keep our service families working correctly. Currently, we are mostly interested in guarantees on the subset of all products within the product line that we actually have in use. As this is a limited set in comparison to the theoretical set of all possible variants, we can specify, implement and test these single services following well-established industry-standard tools and techniques. Therefore, we do not see the risk and costs for a paradigm shift justified in the context of microservice SPLs, as similar observed by Horcas et al. [22].

\subsection{Platform Packaging}

In context of platform packaging (cf. 4.3), concepts of SPLE seem promising to us, as benefits have dependencies between each other and service packages can span multiple services across developer teams. However, as far as we are aware of SPLE techniques, SPLE aims to follow a top-down approach while we potentially require a bottom-up approach [12, 18, 28, 29, 44]. Namely, SPLE treats the variability as first class citizen and aims to construct products out of the variability space within the variability model, effectively leading to feature-aware programming concepts. We will not enforce non-standard programming concepts to be applied. However, we require benefit-related artifacts to be managed. For instance, release notes in services will span multiple benefits, and must be projected to the subset of selected benefits within the particular service package that is used by a client. In the future, this decision might depend on keeping the current structure of our service packages, or breaking them down to the granularity of benefits. Providing a fixed set of service packages each containing a predefined set of benefits reduces complexity and enables us to specify and test service packages similarly to our proceedings for service family SPLs. However, should we at some point in time decide to operate at granularity of benefits, or perform up-sells by benefits rather than in terms of packages, then we might require a formal analysis to enable a managed product configuration services.

\section{SUMMARY}

It surprises that SPLE techniques, such the paradigm of FOP, still seem to wait for their moment to shine across industries. To close this gap, we provide insights into our practice, judging SPLE under different use cases, and elaborate where we think it will shine for us. We start with an introduction to our platform by providing information about our company, mission, technology stack and micro service classes. Afterwards, we share our techniques on configuration management by example for static and dynamic configurations, and compile-time configurations. Based on these techniques, we show how we manage and implement variability in our micro service families by sharing our concepts for specification, implementation, building, testing and deploying services and service families. Here, 
we also provide insights into our platform packaging strategy. Finally, we discuss SPLE for services and for packaging. We conclude that we are good to go with industry-standard techniques for micro service SPLs. The variability modeling and analysis advantages within SPLE is promising for our packaging strategy, though.

\section{REFERENCES}

[1] Mustafa Al-Hajjaji, Jens Meinicke, Sebastian Krieter, Reimar Schröter, Thomas Thüm, Thomas Leich, and Gunter Saake. 2016. Tool Demo: Testing Configurable Systems with FeatureIDE. In Proceedings of the 2016 ACM SIGPLAN International Conference on Generative Programming: Concepts and Experiences. 173-177.

[2] Michał Antkiewicz, Kacper Bak, Alexandr Murashkin, Rafael Olaechea, Jia Hui Liang, and Krzysztof Czarnecki. 2013. Clafer Tools for Product Line Engineering. In Proceedings of the 17th international software product line conference co-located workshops. 130-135.

[3] S Apel, D Batory, C Kästner, and G Saake. [n.d.]. Feature-Oriented Software Product Lines: Concepts and Implementation. URL http://www. springer. com/computer/swe/book/978-3-642-37520-0 ([n. d.]).

[4] Sven Apel, Don Batory, Christian Kästner, and Gunter Saake. 2013. Software Product Lines. In Feature-Oriented Software Product Lines. Springer, 3-15.

[5] Sven Apel, Christian Kastner, and Christian Lengauer. 2009. FeatureHouse Language-Independent, Automated Software Composition. In 2009 IEEE 31st International Conference on Software Engineering. IEEE, 221-231.

[6] Inmaculada Ayala, Mercedes Amor, and Lidia Fuentes. 2012. An Agent Platform for Self-Configuring Agents in the Internet of Things. In Third International Workshop on Infrastructures and Tools for Multiagent Systems, ITMAS. 65-78.

[7] Christian Batory, Lengauer Don, and Charles Consel Martin Odersky. 2004 Domain-Specific Program Generation. Springer.

[8] Don Batory. 2004. Feature-Oriented Programming and the AHEAD Tool Suite. In Proceedings. 26th International Conference on Software Engineering. IEEE, 702-703.

[9] Don Batory. 2005. Feature Models, Grammars, and Propositional Formulas. In International Conference on Software Product Lines. Springer, 7-20.

[10] David Benavides, Sergio Segura, Pablo Trinidad, and Antonio Ruiz-Cortés. 2005. Using Java CSP Solvers in the Automated Analyses of Feature Models. In International Summer School on Generative and Transformational Techniques in Software Engineering. Springer, 399-408.

[11] David Benavides, Pablo Trinidad, and Antonio Ruiz-Cortés. 2005. Automated Reasoning on Feature Models. In International Conference on Advanced Information Systems Engineering. Springer, 491-503.

[12] Fernando Benbassat, Paulo Borba, and Leopoldo Teixeira. 2016. Safe Evolution of Software Product Lines: Feature Extraction Scenarios. In 2016 X Brazilian Symposium on Software Components, Architectures and Reuse (SBCARS). IEEE, $11-20$.

[13] Fabian Benduhn, Reimar Schröter, Andy Kenner, Christopher Kruczek, Thomas Leich, and G ANDSAAKE. 2016. Migration from Annotation-Based to Composition-Based Product Lines: Towards a Tool-Driven Process. In Proc. Conf. Advances and Trends in Software Engineering (SOFTENG). IARIA. 102-109.

[14] Benjamin Benni, Philippe Collet, Guilhem Molines, Sébastien Mosser, and AnneMarie Pinna-Déry. 2018. Teaching DevOps at the Graduate Level. In International Workshop on Software Engineering Aspects of Continuous Development and New Paradigms of Software Production and Deployment. Springer, 60-72.

[15] Francis Bordeleau, Bran Selic, et al. 2005. A Domain Model for Dynamic System Reconfiguration. In International Conference on Model Driven Engineering Languages and Systems. Springer, 553-567.

[16] Paul Clements and Linda Northrop. 1999. A Framework for Software Product Line Practice-Version 1.0, Software Engineering Institute.

[17] Krzysztof Czarnecki and Ulrich W Eisenecker. 2000. Generative Programming. (2000).

[18] Jean-Marc Davril, Edouard Delfosse, Negar Hariri, Mathieu Acher, Jane ClelandHuang, and Patrick Heymans. 2013. Feature Model Extraction from Large Collections of Informal Product Descriptions. In Proceedings of the 2013 9th foint Meeting on Foundations of Software Engineering. 290-300.

[19] Christian Dietrich, Reinhard Tartler, Wolfgang Schröder-Preikschat, and Daniel Lohmann. 2012. A Robust Approach for Variability Extraction from the Linux Build System. In Proceedings of the 16th International Software Product Line Conference-Volume 1. 21-30.

[20] Critina Gacek and Michalis Anastasopoules. 2001. Implementing Product Line Variabilities. In Proceedings of the 2001 symposium on Software reusability: putting software reuse in context. 109-117.

[21] Florian Heidenreich, Pablo Sanchez, Joao Santos, Steffen Zschaler, Mauricio Alferez, Joao Araujo, Lidia Fuentes, Uirá Kulesza, Ana Moreira, and Awais Rashid. 2010. Relating Feature Models to Other Models of a Software Product Line. In Transactions on aspect-oriented software development VII. Springer, 69-114.

[22] Jose-Miguel Horcas, Mónica Pinto, and Lidia Fuentes. 2019. Software Product Line Engineering: a Practical Experience. In Proceedings of the 23rd International
Systems and Software Product Line Conference-Volume A. 164-176.

[23] Kyo C Kang, Sholom G Cohen, James A Hess, William E Novak, and A Spencer Peterson. 1990. Feature-Oriented Domain Analysis (FODA) Feasibility Study. Technical Report. Carnegie-Mellon Univ Pittsburgh Pa Software Engineering Inst.

[24] Christian Kästner and Sven Apel. 2008. Integrating Compositional and Annotative Approaches for Product Line Engineering. In Proc. GPCE Workshop on Modularization, Composition and Generative Techniques for Product Line Engineering. 35-40.

[25] Sebastian Krieter, Marcus Pinnecke, Jacob Krüger, Joshua Sprey, Christopher Sontag, Thomas Thüm, Thomas Leich, and Gunter Saake. 2017. FeatureIDE: Empowering Third-Party Developers. In Proceedings of the 21st International Systems and Software Product Line Conference-Volume B. 42-45.

[26] Jacob Krüger, Thorsten Berger, and Thomas Leich. 2019. Features and How to Find Them: a Survey of Manual Feature Location. Software Engineering for Variability Intensive Systems (2019), 153-172.

[27] Jacob Krüger, Gül Çalıklı, Thorsten Berger, Thomas Leich, and Gunter Saake. 2019. Effects of Explicit Feature Traceability on Program Comprehension. In Proceedings of the 2019 27th ACM Foint Meeting on European Software Engineering Conference and Symposium on the Foundations of Software Engineering. 338-349.

[28] Jacob Krüger, Wolfram Fenske, Jens Meinicke, Thomas Leich, and Gunter Saake. 2016. Extracting Software Product Lines: a Cost Estimation Perspective. In Proceedings of the 20th International Systems and Software Product Line Conference. 354-361.

[29] Thomas Kühn and Walter Cazzola. 2016. Apples and Oranges: Comparing TopDown and Bottom-Up Language Product Lines. In Proceedings of the 20th International Systems and Software Product Line Conference. 50-59.

[30] Robyn Lutz. 2007. Survey of Product-Line Verification and Validation Techniques. Technical Report. Pasadena, CA: Jet Propulsion Laboratory, National Aeronautics and Space ....

[31] Jens Meinicke, Thomas Thüm, Reimar Schröter, Sebastian Krieter, Fabian Benduhn, Gunter Saake, and Thomas Leich. 2016. FeatureIDE: Taming the Preprocessor Wilderness. In 2016 IEEE/ACM 38th International Conference on Software Engineering Companion (ICSE-C). IEEE, 629-632.

[32] Jens Meinicke, Chu-Pan Wong, Christian Kästner, Thomas Thüm, and Gunter Saake. 2016. On Essential Configuration Complexity: Measuring Interactions in Highly-Configurable Systems. In Proceedings of the 31st IEEE/ACM International Conference on Automated Software Engineering. 483-494.

[33] Torsten Nelson, Donald Cowan, and Paulo Alencar. 2001. Supporting Formal Verification of Crosscutting Concerns. In International Conference on Metalevel Architectures and Reflection. Springer, 153-169.

[34] Hector Perez-Morago, Ruben Heradio, David Fernandez-Amoros, Roberto Bean, and Carlos Cerrada. 2015. Efficient Identification of Core and Dead Features in Variability Models. IEEE Access 3 (2015), 2333-2340.

[35] K. Pohl, Günter Böckle, and F. V. Linden. 2005. Software Product Line Engineering - Foundations, Principles, and Techniques.

[36] Dhanji R Prasanna. 2009. Dependency Injection. Manning.

[37] Clément Quinton, Sébastien Mosser, Carlos Parra, and Laurence Duchien. 2011. Using Multiple Feature Models to Design Applications for Mobile Phones. In Proceedings of the 15th International SPLC, Volume 2. 1-8.

[38] Sandro Schulze, Sven Apel, and Christian Kästner. 2010. Code Clones in FeatureOriented Software Product Lines. ACM SIGPLAN Notices 46, 2 (2010), 103-112.

[39] Daniel Strüber, Vlad Acrețoaie, and Jennifer Plöger. 2018. Clone Detection for Rule-Based Model Transformation Languages. Software Engineering und Software Management 2018 (2018).

[40] Daniel Strüber, Julia Rubin, Thorsten Arendt, Marsha Chechik, Gabriele Taentzer, and Jennifer Plöger. 2018. Variability-Based Model Transformation: Formal Foundation and Application. Formal Aspects of Computing 30, 1 (2018), 133-162.

[41] Jing Sun, Hongyu Zhang, Yuan Fang, and Li Hai Wang. 2005. Formal Semantics and Verification for Feature Modeling. In 10th IEEE International Conference on Engineering of Complex Computer Systems (ICECCS'05). IEEE, 303-312.

[42] Thomas Thüm, Sven Apel, Christian Kästner, Ina Schaefer, and Gunter Saake. 2014. A Classification and Survey of Analysis Strategies for Software Product Lines. ACM Computing Surveys (CSUR) 47, 1 (2014), 1-45.

[43] Thomas Thüm, Christian Kästner, Fabian Benduhn, Jens Meinicke, Gunter Saake, and Thomas Leich. 2014. FeatureIDE: An Extensible Framework for FeatureOriented Software Development. Science of Computer Programming 79 (2014), $70-85$.

[44] Edoardo Vacchi, Walter Cazzola, Benoit Combemale, and Mathieu Acher. 2014. Automating Variability Model Inference for Component-Based Language Implementations. In Proceedings of the 18th International Software Product Line Conference-Volume 1.167-176.

[45] Frank J Van der Linden, Klaus Schmid, and Eelco Rommes. 2007. Software Product Lines in Action: the Best Industrial Practice in Product Line Engineering. Springer Science \& Business Media.

[46] Jinshui Wang, Xin Peng, Zhenchang Xing, and Wenyun Zhao. 2011. An Exploratory Study of Feature Location Process: Distinct Phases, Recurring Patterns, and Elementary Actions. In 2011 27th IEEE International Conference on Software Maintenance (ICSM). IEEE, 213-222. 